Pacific Journal of Mathematics

HOMOMORPHISMS AND SUBALGEBRAS OF 


\title{
HOMOMORPHISMS AND SUBALGEBRAS OF MULTIALGEBRAS
}

\author{
H. E. PicketT
}

\begin{abstract}
We shall prove that the homomorphisms and subalgebras of a multialgebra that can be studied naturally through its latticeordered representation as an ordinary algebra are limited to its ideal homomorphisms and Birkhoff subalgebras. However, these form a very limited subclass of the interesting homomorphisms and subalgebras. Of nearly equal importance, for example, are the co-ideal homomorphisms, which arise naturally in (say) groups from left coset decompositions by nonnormal subgroups. To emphasize the special nature of ideal homomorphisms, the class of multiquasigroups is introduced, for which every regular mapping qualifies as a homomorphism. We show in general that ideal (co-ideal) homomorphisms correspond to equivalence relations which we call ideals (co-ideals), and the relationship between ideals, co-ideals, and coset decompositions in multiquasigroups is delineated.
\end{abstract}

The class of algebraic systems known as multialgebras [5], including its several specializations, e.g., multigroups $[6,7,13,14,15]$, multirings [10], multilattices $[1,2]$, etc., seems eminently suited to the study of many problems. For example, multigroups have been used by Marty [9] to obtain results in the theory of rational functions of a complex variable, by Krasner [8] in class field theory, and by Prenowitz $[13,14,15]$ in geometry. Multilattices have been used by Benado [1] to extend a result of Birkhoff [3, p. 149] relating to the relativistic wave equation. Nevertheless, there has been pointed out by several authors (Bruck [4], Dresher and Ore [7], Prenowitz [15]) a representation every multialgebra enjoys as a lattice-ordered complete atomic algebra, and Bruck [4, p. 42] has suggested that multialgebras are studied most appropriately from this point of view. Our intent is to prove, by theorem and example, that this is an inordinately modest conception of the richness of multivalued systems.

Indeed, we shall prove (Theorem 2) that the homomorphisms of a multialgebra that can be studied naturally through the induced homomorphisms of its lattice-ordered representation are limited to certain ideal homomorphisms corresponding to a class of equivalence relations on $X$ which we call ideals. ${ }^{1}$ We shall also show that if $M$

1 The ideals and co-ideals introduced here are a specialization of a much broader concept occurring in the study of relational algebras. The concept of homomorphism introduced in $\$ 2$ is in fact inadequately discriminating, though it serves to justify the above thesis, and it is the author's intent to present in another paper a more thorough analysis of this concept, as it applies in relational algebras. 
is an ordinary algebra, i.e., single valued, then its ideal homomorphs are ordinary algebras. But, one of the more interesting examples of a multigroup homomorphism is the mapping of a group, conceived of as multigroup, onto its left coset decomposition by a nonnormal subgroup. The homomorph thus obtained is always multivalued (cf., Dresher and Ore [7, p. 712]). This possibilty properly embeds the class of groups in the class of multigroups.

In Theorem 3 we prove a result about subalgebras similar to the stated result for homomorphisms. The subalgebras involved we call Birkhoff subalgebras. Benado [2] has invoked a much weaker concept. In $\S 4$ we introduce the concept of multiquasigroup and obtain there certain results relating ideals, co-ideals, and coset decompositions. For example, we find that the equivalence relation determined by a left coset decomposition in a multiquasigroup satisfying the left associative law is a co-ideal. Theorem 9 generalizes a result of Birkhoff. $\S 5$ contains several illustrative examples.

2. Homomorphisms of multialgebras. A multialgebra is a system $M=\left(X, f_{1}, f_{2}, \cdots\right)$, where each primitive operation $f_{i}$ is a function on $X^{\left(k_{i}\right)}$ to the family of nonempty subsets of $X$. That is, given $x_{1}, \cdots, x_{k_{i}}$ in $X, f_{i}\left(x_{1}, \cdots, x_{k_{i}}\right)=A \subseteq X, A \neq \varnothing$. The sequence of integers $k_{1}, k_{2}, \cdots$ is the species, or order type, of the algebra. A multialgebra may also be viewed as a relational system with relations $f_{i}$ of rank $k_{i}+1$. A composition of these functions, say $f_{i}\left(f_{j}\left(x_{1}, \cdots, x_{k}\right)\right.$, $\left.y_{2}, \cdots, y_{k_{i}}\right)$, is defined to be the function whose values are

$$
\bigcup_{z} f_{i}\left(z, y_{2}, \cdots, y_{k_{i}}\right) \text {, }
$$

where $z$ runs over the elements of $f_{j}\left(x_{1}, \cdots, x_{k_{j}}\right)$. There is a natural one-one correspondence between ordinary algebras, and those multialgebras in which every set $A$ holds exactly one element.

In the sequel we shall denote a vector $\left(x_{1}, \cdots, x_{k}\right)$ by $\boldsymbol{x}$ and write $f(x)$ in place of $f\left(x_{1}, \cdots, x_{k}\right)$. We shall also adopt a useful notation of Prenowitz [14], and write $f(\boldsymbol{x}) \approx a(\operatorname{read} f(\boldsymbol{x})$ meets $a)$ to mean $a \in f(\boldsymbol{x})$. This notation he extends to sets, writing $A \approx B$ to mean $A \cap B \neq \varnothing$. The relation is clearly reflexive and symmetric on the family of nonempty subsets of $X$, but not transitive. If $h$ is a manyone map from $X$ to $X^{*}$ we let $h(A)$ denote $\{h(a) \mid a \in A\}$, and $h(\boldsymbol{x})$ denote $\left(h\left(x_{1}\right), \cdots, h\left(x_{k}\right)\right)$ in the customary fashion. Also $h^{-1}\left(a^{*}\right)=$ $\left\{a \mid h(a)=a^{*}\right\}$, and $h^{-1}\left(\boldsymbol{x}^{*}\right)=\left\{\boldsymbol{x} \mid h(\boldsymbol{x})=\boldsymbol{x}^{*}\right\}$, where $h^{-1}$ denotes the inverse of the map $h$.

A many-one map $h$ from a multialgebra $M$ onto a second multialgebra $M^{*}$ of the same species is a (regular) homomorphism (Pickert [11]) if and only if the following two conditions are met 
for each pair of corresponding primitive functions, $f, f^{*}$ :

1. $f(\boldsymbol{x}) \approx a$ implies $f^{*}(h(\boldsymbol{x})) \approx h(a)$,

2. $f^{*}\left(\boldsymbol{x}^{*}\right) \approx a^{*}$ implies $f\left(h^{-1}\left(x^{*}\right)\right) \approx h^{-1}\left(a^{*}\right)$.

A one-one map that is a homomorphism in both directions is an isomorphism. Condition 1 is equivalent to either of the following:

$1^{\prime} . \quad f^{*}(h(\boldsymbol{x})) \supseteqq h(f(\boldsymbol{x}))$,

$1^{\prime \prime} . \quad f\left(h^{-1}\left(x^{*}\right)\right) \subseteq h^{-1}\left(f^{*}\left(x^{*}\right)\right)$.

If equality holds in either of $1^{\prime}$ or $1^{\prime \prime}$ then 2 is satisfied. If equality holds in $1^{\prime}$ for every pair $f, f^{*}$ we shall say $h$ is an ideal homomorphism. This attractive generalization from the concept of homomorphism for ordinary algebras has been adopted by several authors (Bruck [4], Prenowitz [13]). If equality holds for $1^{\prime \prime}$ for every pair $f, f^{*}$ we shall say $h$ is a co-ideal homomorphism. It can be shown that if $f\left(a_{1}, \cdots, a_{n}\right) \subseteq g\left(a_{1}, \cdots, a_{n}\right)$ is a compositional tautology in $M$, i.e., is valid for every substitution for $a_{1}, \cdots, a_{n}$, then the same compositional tautology is valid in both ideal and co-ideal homomorphs.

An equivalence relation $E$ on $X$ is an ideal (or ideal congruence relation) for $M$ if and only if it satisfies for every primitive function $f$ of $M$ the condition: $f(x) \approx a$ and $(\boldsymbol{x}, \boldsymbol{y}) \in E$ imply, for some $b$, that $f(\boldsymbol{y}) \approx b$ and $(a, b) \in E$. Here $(\boldsymbol{x}, \boldsymbol{y}) \in E$ means $\left(x_{i}, y_{i}\right) \in E, i=1, \cdots, k$. $E$ is a co-ideal for $M$ if and only if it satisfies for every primitive function $f$ the condition: $f(\boldsymbol{x}) \approx a$ and $(a, b) \in E$ imply, for some $\boldsymbol{y}$, that $f(\boldsymbol{y}) \approx b$ and $(\boldsymbol{x}, \boldsymbol{y}) \in E$. We note that if $f$ is a single-valued function, everywhere defined, then the condition defining ideals is equivalent to the condition: $(\boldsymbol{x}, \boldsymbol{y}) \in E$ implies $(f(\boldsymbol{x}), f(\boldsymbol{y})) \in E$. Hence, if $M$ is an ordinary algebra, $E$ is an ideal if and only if it is a congruence relation for $M$ (Birkhoff [3, p. vii]).

Corresponding to any equivalence relation $E$ on $X$ is a partition of $X$ into pairwise disjoint, nonempty, subsets. We denote this family of subsets by $X / E$, and that member of the family to which an element $x$ belongs by $x / E$. We also write $A / E$ to denote $\{a / E \mid a \in A\}$ and $\boldsymbol{x} / E$ to denote $\left(x_{1} / E, \cdots, x_{k} / E\right)$. We define $f / E$ to be that function $f^{*}$ on $(X / E)^{k}$ to $X / E$ such that $f^{*}\left(\boldsymbol{x}^{*}\right)=\bigcup\{A / E \mid$ for some $\boldsymbol{x}, f(\boldsymbol{x})=A$ and $\left.\boldsymbol{x}^{*}=\boldsymbol{x} / E\right\}$. Finally, $M / E=\left(X / E, f_{1} / E, f_{2} / E, \cdots\right)$ is the factor algebra determined by $E$. This is identical to the factor system obtained if $M$ is regarded as a relational system (cf. Pickett [12]). If $M$ is an ordinary algebra then $M / E$ is an ordinary algebra if and only if $E$ is a congruence relation. The map $p: x \rightarrow x / E$ is the partition map determined by $E$.

THEOREM 1. If $h: M \rightarrow M^{*}$ is an ideal (co-ideal) homomorphism then $E=\{(x, y) \mid h(x)=h(y)\}$ is an ideal (co-ideal) of $M$. Conversely, if $E$ is an ideal (co-ideal) of $M$ then the partition map $p: M \rightarrow M / E$ 
is an ideal (co-ideal) homomorphism. Moreover, the map $p\left(h^{-1}\left(x^{*}\right)\right)$ $i s$ an isomorphism between $M^{*}$ and the factor algebra $M / E$.

Proof. The verification that $E$ is an equivalence relation is immediate. Now let $f, f^{*}$ be corresponding functions of $M$ and its ideal homomorph $M^{*}$, respectively. If $(\boldsymbol{x}, \boldsymbol{y}) \in E$ then $h(\boldsymbol{x})=h(\boldsymbol{y})$ and hence

$$
h(f(\boldsymbol{x}))=f^{*}(h(\boldsymbol{x}))=f^{*}(h(\boldsymbol{y}))=h(f(\boldsymbol{y})) .
$$

One concludes that if $f(\boldsymbol{x}) \approx a$ and $(\boldsymbol{x}, \boldsymbol{y}) \in E$ then for some $b, f(\boldsymbol{y}) \approx b$ and $h(a)=h(b)$, i.e., $(a, b) \in E$. Therefore $E$ is an ideal for $M$. For the converse let $f^{\prime}=f / E$. If $f(\boldsymbol{x}) \approx a$ then $f^{\prime}(\boldsymbol{x} / E) \approx a / E$ and hence $p(f(\boldsymbol{x})) \subseteq f^{\prime}(p(\boldsymbol{x}))$. Suppose $f^{\prime}(p(\boldsymbol{x})) \approx b^{\prime}$. Then for some $\boldsymbol{y}$, where $p(\boldsymbol{y})=p(\boldsymbol{x})$, and some $b$, where $p(b)=b^{\prime}$, we have $f(\boldsymbol{y}) \approx b$. Hence, $(\boldsymbol{x}, \boldsymbol{y}) \in E$, and $f(\boldsymbol{y}) \approx b$, and because $E$ is an ideal, then for some $a$, $f(\boldsymbol{x}) \approx a$ and $(a, b) \in E$. Thus $p(a)=b^{\prime}$, implying $f^{\prime}(p(\boldsymbol{x})) \subseteq p(f(\boldsymbol{x}))$. This proves $p$ is an ideal homomorphism. The corresponding proofs for co-ideals are analogous, and the stated isomorphism is easily shown.

We now show how to construct the lattice ordered representation discussed in the introduction. Let $M=\left(X, f_{1}, f_{2}, \cdots\right)$ be a multialgebra, and let $\|X\|_{0}$ be the family of all nonempty subsets of $X$. Corresponding to each primitive function $f_{i}$ of $M$ we construct a single valued function $F_{i}$ on $\|X\|_{0}$ in the following way. If $X_{1}, \cdots, X_{k_{i}}$ are in $\|X\|_{0}$ then $F_{i}\left(X_{1}, \cdots, X_{k_{i}}\right)=\bigcup f_{i}\left(x_{1}, \cdots, x_{k_{i}}\right)$, where the union is over all $x_{j} \in X_{j}, j=1, \cdots, k_{i}$. Under the ordering of set inclusion $\|X\|_{0}$ forms a partially ordered set, and if $X_{1} \subseteq Y_{1}, \cdots, X_{k_{i}} \subseteq Y_{k_{i}}$, then

$$
F_{i}\left(X_{1}, \cdots, X_{k_{i}}\right) \subseteq F_{i}\left(Y_{1}, \cdots, Y_{k_{i}}\right)
$$

This makes the algebra $\left(\|X\|_{0}, F_{1}, F_{2}, \cdots\right)$ a partially ordered algebra, for which the set $\|X\|_{0}$ may be classified as a complete atomic lattice (ignoring the lack of a minimal element). Conversely, given such an algebra the space may be conceived as the set of nonempty subsets of some set $X$, and $M$ may be retrieved by defining $f_{i}\left(x_{1}, \cdots, x_{k_{i}}\right)=$ $F_{i}\left(\left\{x_{1}\right\}, \cdots,\left\{x_{k_{i}}\right\}\right)$. Our discussion has followed Bruck [4, p. 42].

Suppose there is given a homomorphism $h$ of a multialgebra $\left(X, f_{1}, f_{2}, \cdots\right)$ onto a multialgebra $\left(Y, g_{1}, g_{2}, \cdots\right)$ of the same species. Construct the algebras $\left(\|X\|_{0}, F_{1}, F_{2}, \cdots\right)$ and $\left(\|Y\|_{0}, G_{1}, G_{2}, \cdots\right)$ by the above method. The map $h$ of $X$ onto $Y$ induces a map $H$ of $\|X\|_{0}$ onto $\|Y\|_{0}$ defined by $H(A)=\{h(a) \mid a \in A\}$, for each $A$ in $\|X\|_{0}$. G. Pickert [11, p. 379] has queried, under what conditions on $h$ is the induced map $H$ a homomorphism, i.e., an ordinary or algebraic homomorphism? We answer this with the following theorem. 
THEOREM 2. A necessary and sufficient condition that the induced map $H$ be an ordinary homomorphism is that $E=\{(x, y) \mid h(x)=h(y)\}$ should be an ideal for $\left(X, f_{1}, f_{2}, \cdots\right)$.

Proof. We shall use $X$ to denote the vector $\left(X_{1}, \cdots, X_{k}\right)$, where $X_{1}, \cdots, X_{k}$ are in $\|X\|_{0}$. The induced map $H$ determines an equivalence relation $\boldsymbol{E}=\{(A, B) \mid H(A)=H(B)\}$, and $H$ is an algebraic homomorphism if and only if $E$ is an algebraic congruence relation, that is, satisfies the condition

(I) $(\boldsymbol{X}, \boldsymbol{Y}) \in \boldsymbol{E}$ implies $(F(\boldsymbol{X}), F(\boldsymbol{Y})) \in \boldsymbol{E}$ for each primitive function $F$ of $\left(\|X\|_{0}, F_{1}, F_{2}, \cdots\right)$. We note further that $(A, B) \in \boldsymbol{E}$ if and only if $a \in A$ implies, for some $b \in B$, that $(a, b) \in E$, and $b \in B$ implies, for some $a \in A$, that $(a, b) \in E$. Recall that $E$ is an ideal for $\left(X, f_{1}, f_{2}, \ldots\right)$ if and only if it satisfies the condition

(II) $f(\boldsymbol{x}) \approx a$ and $(\boldsymbol{x}, \boldsymbol{y}) \in E$ imply, for some $b$, that $f(\boldsymbol{y}) \approx b$ and $(a, b) \in E$,

for each primitive function $f$. We must show that $E$ is an ideal if and only if $\boldsymbol{E}$ is a congruence relation. (I) implies (II), for let $(\boldsymbol{x}, \boldsymbol{y}) \in E$ and $f(\boldsymbol{x}) \approx a$. Define $X_{i}=\left\{a_{i}\right\}, Y_{i}=\left\{y_{i}\right\}, i=1, \cdots, k$. Then $(\boldsymbol{X}, \boldsymbol{Y}) \in \boldsymbol{E}$ and $F(\boldsymbol{X}) \approx a$. In view of condition (I), and the comment following, there exists $b$ such that $F(\boldsymbol{Y}) \approx b$ and $(a, b) \in E$. Then $f(\boldsymbol{y}) \approx b$ and $E$ is an ideal. (II) implies (I), for let $F(\boldsymbol{X}) \approx a$ and choose $x_{1} \in X_{1}, \cdots, x_{k} \in X_{k}$ so that $f(x) \approx a$. For a $Y$ such that $(\boldsymbol{X}, \boldsymbol{Y}) \in \boldsymbol{E}$ choose $y_{1} \in Y_{1}, \cdots, y_{k} \in Y_{k}$ so that $(\boldsymbol{x}, \boldsymbol{y}) \in E$. By II there exists $b$ such that $f(\boldsymbol{y}) \approx b$ and $(a, b) \in E$. But then $F(\boldsymbol{Y}) \approx b$, so that $(F(\boldsymbol{X}), F(\boldsymbol{Y})) \in \boldsymbol{E}$, and hence $\boldsymbol{E}$ is a congruence relation.

In view of Theorem 1 this result may also be stated as follows: $H$ is an ordinary homomorphism if and only if $h$ is an ideal homomorphism. This leads to the following corollary. If $h$ is not an ideal homomorphism the factor algebra $\left(\|X\|_{0}, F_{1}, F_{2}, \cdots\right) / E$ is multivalued. For the only equivalence relations that preserve singlevaluedness are the congruence relations. Hence, only by restricting one's attention to ideal homomorphism can multivalued systems be avoided. We shall try to indicate in $\S 4$ and $\S 5$ why this restriction is undesirable.

3. Submultialgebras. A submultialgebra of $\left(X, f_{1}, f_{2}, \cdots\right)$ is, for most of the generic types, understood to be a system $\left(Y, g_{1}, g_{2}, \cdots\right)$ of the same species, where $Y \subseteq X$, and for $y_{1}, \cdots, y_{k_{i}}$ in $Y, g_{i}\left(y_{1}, \cdots, y_{k_{i}}\right)=$ $f_{i}\left(y_{1}, \cdots, y_{k_{i}}\right) \subseteq Y$. This seems to be a natural definition to employ, reflecting as it does Birkhoff's [3, p. vii] dictum that a subalgebra should "include every algebraic combination of its own elements". But, just as for ordinary algebras, one must not expect submulti- 
algebras to possess all of the defining properties of the parent algebra. ${ }^{2}$ We refer to these subsystems as Birkhoff subalgebras.

A weaker conception of subalgebra, sometimes of use, has been given by Benado [2, p. 313]. He defines $\left(Y, g_{1}, g_{2}, \cdots\right)$ to be a subalgebra if $g_{i}\left(y_{1}, \cdots, y_{k_{i}}\right)=Y \cap f_{i}\left(y_{1}, \cdots, y_{k_{i}}\right) \neq \varnothing$. It is clear that every Birkhoff subalgebra is a subalgebra under this definition. As in the case of ordinary algebras the family of all Birkhoff subalgebras is a lattice. With certain restrictions the subalgebras defined by Benado form a multilattice.

Suppose, now, that there is given a multialgebra $\left(X, f_{1}, f_{2}, \cdots\right)$, and a second multialgebra $\left(Y, g_{1}, g_{2}, \cdots\right)$, which is a subalgebra in the sense of Benado. Carry out the construction of the latticeordered representations of these systems, yielding $\left(\|X\|_{0}, F_{1}, F_{2}, \cdots\right)$ and $\left(\|Y\|_{0}, G_{1}, G_{2}, \cdots\right)$, respectively. Following the example of Pickert we ask, what restriction must be placed on the set $Y$ in order that the second representation should be a subalgebra of the first? The answer is significant, but hardly surprising.

THEOREM 3. A necessary and sufficient condition for (\|Y $Y$ o, $\left.G_{1}, G_{2}, \cdots\right)$ to be a subalgebra of $\left(\left\|X_{0}\right\|, F_{1}, F_{2}, \cdots\right)$ is that $\left(Y, g_{1}, g_{2}, \cdots\right)$ should be a Birkhoff subalgebra of $\left(X, f_{1}, f_{2}, \cdots\right)$.

Proof. If $\left(\|Y\|_{0}, G_{1}, G_{2}, \cdots\right)$ is a subalgebra then, for each $i$, given $y_{1}, \cdots, y_{k_{i}}$ in $Y$,

$$
\begin{aligned}
f_{i}\left(y_{1}, \cdots, y_{k_{i}}\right) & =F_{i}\left(\left\{y_{1}\right\}, \cdots,\left\{y_{k_{i}}\right\}\right) \\
& =G_{i}\left(\left\{y_{1}\right\}, \cdots,\left\{y_{k_{i}}\right\}\right) \subseteq Y,
\end{aligned}
$$

so $\left(Y, g_{1}, g_{2}, \cdots\right)$ is a Birkhoff subalgebra. Conversely, if $\left(Y, g_{1}, g_{2}, \cdots\right)$ is a Birkhoff subalgebra, then given $Y_{1}, \cdots, Y_{k_{i}}$ in $\|Y\|_{0}$,

$$
\begin{aligned}
G_{i}\left(Y_{1}, \cdots, Y_{k_{i}}\right) & =\bigcup g_{i}\left(y_{1}, \cdots, y_{k_{i}}\right) \\
& =\bigcup f_{i}\left(y_{1}, \cdots, y_{k_{i}}\right)=F_{i}\left(Y_{1}, \cdots, Y_{k_{i}}\right),
\end{aligned}
$$

where the unions run over all $y_{j} \in Y_{j}, j=1, \cdots, k_{i}$. Hence $\left(\|Y\|_{0}\right.$, $\left.G_{1}, G_{2}, \cdots\right)$ is a subalgebra.

We conclude that the only subsystems of multialgebras that can be studied naturally through the lattice-ordered representation are the Birkhoff subalgebras.

4. Multiquasigroups. A multigroupoid (Bruck [4]) $M=(X, \cdot)$ is a multialgebra with a single binary operation. We will call a multi-

${ }^{2}$ For example, the system $(Z,+)$ of integers under addition is a group, but the set of positive integers, though closed under all additive combinations, is only a semigroup. 
groupoid a multiquasigroup $p^{3}$ if and only if the conditions $a \cdot x \approx b$ and $y \cdot a \approx b$ have solutions for every $a$ and $b$ in $X$. A multigroup (Dresher and Ore [7]) is a multiquasigroup in which the associative law $a \cdot(b \cdot c)=(a \cdot b) \cdot c$ holds. An element 1 of a multigroupoid is a left unit (right unit) if and only if $1 \cdot a \approx a(a \cdot 1 \approx a)$ for every $a$ in $X$, and is a unit if and only if both conditions hold. A multiquasigroup with a unit is a multiloop. In $\S 5$ we give a number of examples illustrating these systems.

The Birkhoff subalgebras of a multiquasigroup are not usually multiquasigroups. This may be overcome by introducing inverse operations $a \cdot b=\{x \mid x \cdot b \approx a\}$ and $a / \cdot b=\{x \mid b \cdot x \approx a\}$. The Birkhoff submultialgebras of the algebra $(X, \cdot, \cdot /, / \cdot)$ correspond to certain submultiquasigroups of $(X, \cdot)$. These have been termed closed by Dresher and Ore, in the case of multigroups, because they contain with every $a$ and $b$ all solutions of $a \cdot x \approx b$ and $y \cdot a \approx b$.

According to our earlier definition a regular homomorphism of a multiquasigroup $M=(X, \cdot)$ onto a multiquasigroup $M^{*}=\left(X^{*}, \circ\right)$ is a many-one map $h$ on $X$ onto $X^{*}$ satisfying

A1. $a \cdot b \approx c$ implies $h(a) \circ h(b) \approx h(c)$,

A2. $a^{*} \circ b^{*} \approx c^{*}$ implies $h^{-1}\left(a^{*}\right) \cdot h^{-1}\left(b^{*}\right) \approx h^{-1}\left(c^{*}\right)$.

We observe that $M^{*}$ need not be specified to be a multiquasigroup, for given $a^{*}, b^{*}$ belonging to $M^{*}$ there are preimages $a, b$ belonging to $M$, and also $x$ and $y$ belonging to $M$, such that $a \cdot x \approx b, y \cdot a \approx b$, so that $a^{*} \circ h(x) \approx b^{*}, h(y) \circ a^{*} \approx b^{*}$, and thus $M^{*}$ is a multiquasigroup. Thus every regular map of $M$ onto $M^{*}$ deserves to be called a multiquasigroup homomorphism. Since there is a one-one correspondence (up to isomorphism) between the regular maps on $M$ and the equivalence relations $E$ on $X$, every factor system $M / E$ should be called a homomorph of $M$.

Let $M=(X, \cdot)$ be a multiquasigroup, and $Y$ a nonempty subset of $X$. A left coset of $Y$ is a set $Y_{a}=Y \cdot a=\{b \mid$ for some $y \in Y, y \cdot a \approx b\}$. $Y$ determines a left coset decomposition of $X$ if and only if

B1. for each $a \in X, Y_{a} \approx a$,

B2. for each $a, b$ in $X$, either $Y_{a}=Y_{b}$ or $Y_{a} \approx Y_{b}$.

The cosets of a left coset decomposition can be given a natural composition as follows. The product $Y_{a} \circ Y_{b}$ of two cosets is the set of all cosets $Y_{c}$ such that for some $a_{1}$ in $Y_{a}$, for some $b_{1}$ in $Y_{b}, a_{1} \cdot b_{1} \approx Y_{c}$. Clearly the multiquasigroup $\left(\left\{Y_{a}, a \in X\right\}, \circ\right)$ is simply the factor algebra $M / E$, where $E$ is the equivalence relation associated with the partition. Also clearly, the mapping $h: a \rightarrow Y_{a}$ is a regular homomorphism of $M$ onto $M / E$. From our present point of view, coset decompositions are important because they provide examples of multialgebra homomor-

${ }^{3}$ Insofar as the author knows, such a concept has not previously been introduced. 
phisms which are not determined by ideals. On the other hand, not all ideals are determined by coset decompositions. Examples to this effect are given in $\S 5$.

If $E$ is an equivalence relation on $X$ let us write $a \equiv b(E)$, meaning $(a, b) \in E . \quad E$ is a left congruence relation for $M$ if and only if there is a subset $Y$ of $X$ such that $a \equiv b(E)$ if and only if for some $x$ in $Y, x \cdot a \approx b$.

THeORem 4. The left coset decompositions of a multiquasigroup $M$ correspond one-one to the left congruence relations for $M$.

Proof. Suppose first that $Y$ determines a left coset decomposition of $X$. Define $a \equiv b(E)$ if and only if $Y_{a} \approx Y_{b}$, i.e., if and only if $Y_{a}=Y_{b}$. If $a \equiv b(E)$, then $b \approx Y_{a}$, so for some $x$ in $Y, x \cdot a \approx b$. If for some $x$ in $Y, x \cdot a \approx b$, then $Y_{a}=Y_{b}$ so $a \equiv b(E)$. Thus $E$ is a left congruence relation. Next suppose $E$ is a left congruence relation for $M$, and let $Y$ be a subset of $X$ with the specified property. Let $a \equiv b(E)$. Then for some $x$ in $Y, x \cdot a \approx b$, so $b \approx Y_{a}$. Hence congruent elements belong to the same coset. Suppose $a \neq \equiv(E)$. Then for no $x$ in $Y$ does $x \cdot a \approx b$, so that $b \approx Y_{a}$. Thus noncongruent elements belong to different cosets.

It is of some interest to characterize those subsets $Y$ giving rise to a left coset decomposition. We observe first that it is necessary and sufficient that $Y$ satisfy, for every $a, b, c$ in $X$, the following three conditions:

C1. $Y \cdot a \approx a$,

C2. $Y \cdot a \approx b$ implies $Y \cdot b \approx a$,

C3. $Y \cdot a \approx b$ and $Y \cdot b \approx c$ imply $Y \cdot a \approx c$.

If $Y \neq \varnothing$ we have for every $a$, for some $b$, that $Y \cdot a \approx b$. Then by $\mathrm{C} 2$ and $\mathrm{C} 3$ we obtain $Y \cdot a \approx a$. If $Y$ is nonvoid a condition equivalent to $\mathrm{C} 2$ and $\mathrm{C} 3$ together is

C4. $Y \cdot a \approx b$ and $Y \cdot c \approx b$ imply $Y \cdot a \approx c$.

Hence if $Y$ is nonvoid $\mathrm{C} 4$ characterizes $Y$. Observe that if $Y_{1}$ and $Y_{2}$ both determine the same left coset decomposition then $Y_{1} \cup Y_{2}$ does also. This implies that to every left coset decomposition there corresponds a unique maximal set determining that decomposition. We call such a set the decomposition set of the decomposition. In general this set need not itself belong to the decomposition. If it does we call the decomposition regular.

The condition $\mathrm{C} 3$ is equivalent to the compositional relation $Y \cdot(Y \cdot a) \cong Y \cdot a$, for every $a$. If the left associative law $a \cdot(b \cdot c) \cong$ $(a \cdot b) \cdot c$ is in effect in $M$, and if $Y$ is any Birkhoff subalgebra, then the condition $\mathrm{C} 3$ is necessarily satisfied, for then 


$$
Y \cdot(Y \cdot a) \subseteq(Y \cdot Y) \cdot a \subseteq Y \cdot a .
$$

In this case, to determine a left coset decomposition $Y$ need only be a Birkhoff subalgebra satisfying C2. Dresher and Ore have, in the case of multigroups, called such subalgebras left reversible.

If $Y$ determines a coset decomposition it is itself a coset if and only if $Y \cdot y=Y$, for every $y$ in $Y$. It is therefore a Birkhoff subalgebra, and also has the property, which we call left inversive, that for every $a, b$ in $Y$ there is a solution in $Y$ of $x \cdot a \approx b$. Conversely, a left inversive subalgebra $Y$ satisfies $Y \cdot y=Y$, for every $y$ in $Y$. In general, $Y$ need not even be a Birkhoff subalgebra, and a necessary and sufficient condition for this is that $Y \cdot y \subseteq Y$ for every $y$ in $Y$, i.e., the cosets $Y_{y}$ must be contained in $Y$. In this case, these cosets are themselves Birkhoff subalgebras, for if $a$ is in $Y$, then

$$
Y_{a} \cdot Y_{a} \subseteq Y \cdot Y_{a}=Y_{a} .
$$

However, if $M$ satisfies the right associative law $(a \cdot b) \cdot c \leqq a \cdot(b \cdot c)$, the decomposition set of a left coset decomposition is a Birkhoff subalgebra, for let $Y$ be such a maximal set, and let $Z=Y \cdot Y \supseteqq Y$. Then $Z \cdot a \approx b$ if and only if $Y \cdot a \approx b$, since clearly $Y \cdot a \approx b$ implies $Z \cdot a \approx b$, and if $Z \cdot a \approx b$, then $b \approx(Y \cdot Y) \cdot a \subseteq Y \cdot(Y \cdot a)=Y \cdot a$. Since $Y$ is maximal, $Z=Y \cdot Y \subseteq Y$.

In order for a left coset decomposition to be regular the decomposition set must be a left inversive Birkhoff subalgebra. If a closed Birkhoff subalgebra $Y$ determines a left coset decomposition, $Y$ is the decomposition set, and the decomposition is regular. For clearly it is left inversive, and if $Z \cdot a=Y \cdot a$, for every $a$, then, for $y$ in $Y, Z \cdot y \subseteq Y$, implying $Z \subseteq Y$. Again, if $M$ has a right unit, and if a Birkhoff subalgebra $Y$ determines a left coset decomposition, then $Y$ is the decomposition set of a regular decomposition, for $Y \cdot 1 \approx y, y$ in $Y$, implies $Y \cdot y \approx 1$, so 1 is in $Y$. Hence $Y \subseteq Y \cdot 1 \subseteq Y$, i.e., $Y=Y \cdot 1$ is a coset. Therefore $Y$ is left inversive. If $Z \cdot a=Y \cdot a$, for every $a$, then $Z \cong Z \cdot 1=Y \cdot 1=Y$, so $Y$ is the maximal set determining the decomposition.

As a corollary to the above remarks we obtain the following result.

THEOREM 5. Let $M$ be a multigroup with a right unit. Then the left coset decompositions correspond one-one to the left reversible, left inversive, Birkhoff submultigroups, and all such decompositions are regular.

In a similar fashion we can discuss right coset decompositions, and simultaneous left and right coset decompositions. If a set $Y$ 
determines left and right coset decompositions which are identical we shall simply refer to a coset decomposition. A necessary and sufficient condition for this to be true is that, for every $a, Y \cdot a=a \cdot Y$. Such subsets have been called normal by Dresher and Ore. A reversible Birkhoff subalgebra is a closed submultiquasigroup, since, e.g., $Y \cdot a \approx$ $b \approx Y$ implies $Y \cdot b \approx a$, and thus $a$ is in $Y$. Moreover, normality and the left conditions $\mathrm{C} 1, \mathrm{C} 2, \mathrm{C} 3$, or $\mathrm{C} 4$ imply the corresponding right conditions. We have the following theorem.

THEOREM 6. The regular coset decompositions of a multiquasigroup $M$ correspond one-one to the nonvoid normal Birkhoff subalgebras satisfying C4. These subalgebras are closed submultiquasigroups. If $M$ satisfies either the left associative law or the right associative law the coset decomposition is always regular and corresponds one-one to its nonvoid normal reversible Birkhoff subalgebras.

We turn now to the question of the relationship between ideals, co-ideals, and left coset decompositions. An ideal for a multigroupoid $M=(X, \cdot)$ is an equivalence relation $E$ on $X$ such that $a \equiv a_{1}(E)$ and $b \equiv b_{1}(E)$ and $a \cdot b \approx c$ imply, for some $c_{1}$, that $a_{1} \cdot b_{1} \approx c_{1}$ and $c \equiv c_{1}(E)$. An equivalence relation $E$ is a co-ideal for $M$ if and only if $a \cdot b \approx c$ and $c \equiv c_{1}(E)$ imply, for some $a_{1}, b_{1}$, that $a \equiv a_{1}$ and $b \equiv b_{1}$ and $a_{1} \cdot b_{1} \approx c_{1}$. In general there will be no left coset decomposition that coincides with $X / E$.

THEOREM 7. Let $Y$ determine a left coset decomposition of a multiquasigroup $M$ and let $E$ be the corresponding left congruence relation. Then

a. $E$ is an ideal if and only if for every $a, b,(Y \cdot a) \cdot(Y \cdot b) \leqq$ $Y \cdot(a \cdot b)$,

b. $E$ is a co-ideal if and only if $(Y \cdot a) \cdot(Y \cdot b) \approx c$ implies $(Y \cdot a) \cdot(Y \cdot b) \supseteqq Y \cdot c$.

Hence,

c. $E$ is both an ideal and a co-ideal if and only if $(Y \cdot a) \cdot(Y \cdot b)=$ $Y \cdot(a \cdot b)$ for every $a, b$.

d. If $E$ is an ideal then $Y \cdot Y$ is a Birkhoff subalgebra and $(Y \cdot Y) \cdot a \approx b$ if and only if $Y \cdot a \approx b$.

Further, if $M$ satisfies the left associative law then $E$ is a co-ideal, and every compositional tautology that holds in $M$ holds in $M / E$. If $M$ is a multigroup and if $Y$ is a normal Birkhoff subalgebra then $E$ is an ideal.

Proof. Suppose $E$ is an ideal. Let $a_{1} \approx Y \cdot a, b_{1} \approx Y \cdot b$, and $c_{1} \approx$ 
$a_{1} \cdot b_{1}$. Then $a \equiv a_{1}(E)$ and $b \equiv b_{1}(E)$ so that, for some $c \equiv c_{1}(E)$, $a \cdot b \approx c$, and $c_{1} \approx Y \cdot c$. Hence $a_{1} \cdot b_{1} \subseteq Y \cdot(a \cdot b)$, implying $(Y \cdot a) \cdot(Y \cdot b) \subseteq$ $Y \cdot(a \cdot b)$. Conversely, let $(Y \cdot a) \cdot(Y \cdot b) \subseteq Y \cdot(a \cdot b)$, for every $a, b$ and let $a \equiv a_{1}(E), b \equiv b_{1}(E)$. Then $(a \cdot b) \subseteq Y \cdot\left(a_{1} \cdot b_{1}\right)$ and hence if $a \cdot b \approx c$ then for some $c_{1} \approx a_{1} \cdot b_{1}, c \approx Y \cdot c_{1}$, showing $E$ is an ideal.

If $E$ is a co-ideal, $(Y \cdot a) \cdot(Y \cdot b) \approx c$, and $c_{1} \approx Y \cdot c$, then there exists $a_{1} \equiv a(E), b_{1} \equiv b(E)$, such that $a_{1} \cdot b_{1} \approx c_{1}$. Hence $(Y \cdot a) \cdot(Y \cdot b) \supseteqq Y \cdot c$. Conversely, if $(Y \cdot a) \cdot(Y \cdot b) \approx c$ implies $(Y \cdot a) \cdot(Y \cdot b) \supseteqq Y \cdot c$, let $c_{1} \equiv c(E)$ and $a \cdot b \approx c$. Then $(Y \cdot a) \cdot(Y \cdot b) \approx c$, so for some $a_{1} \approx Y \cdot a$, some $b_{1} \approx$ $Y \cdot b, a_{1} \cdot b_{1} \approx c_{1}$. Hence $E$ is a co-ideal. If $M$ satisfies the left associative law, and if $(Y \cdot a) \cdot(Y \cdot b) \approx c$, then

$$
(Y \cdot a) \cdot(Y \cdot b)=[Y \cdot(Y \cdot a)] \cdot(Y \cdot b) \supseteqq Y \cdot[(Y \cdot a) \cdot(Y \cdot b)] \supseteqq Y \cdot c,
$$

proving that $E$ is necessarily a co-ideal.

If $E$ is a co-ideal we see that $Y_{c} \approx Y_{a} \circ Y_{b}$ if and only if $c \approx(Y \cdot a) \cdot(Y \cdot b)$. Similarly, $Y_{d} \approx\left[Y_{a} \circ Y_{b}\right] \circ Y_{c}$ if and only if

$$
d \approx[(Y \cdot a) \cdot(Y \cdot b)] \cdot(Y \cdot c)
$$

since $Y_{a} \approx\left[Y_{a} \circ Y_{b}\right] \circ Y_{c}$ if and only if for some $Y_{\epsilon}, Y_{e} \approx Y_{a} \circ Y_{b}$ and $Y_{d} \approx Y_{e} \circ Y_{c}$, which is true if and only if for some $e, e \approx(Y \cdot a) \cdot(Y \cdot b)$ and $d \approx(Y \cdot e) \cdot(Y \cdot c)$ and hence if and only if $d \approx[(Y \cdot a) \cdot(Y \cdot b)] \cdot(Y \cdot c)$. Since every composition is built up from shorter compositions it is easy to give an inductive argument in the same way as above showing that, if $f, f^{*}$ are corresponding compositions in $M, M / E$, then $Y_{a} \approx$ $f^{*}\left(Y_{a_{1}}, \cdots, Y_{a_{n}}\right)$ if and only if $a \approx f\left(Y \cdot a_{1}, \cdots, Y \cdot a_{n}\right)$. It immediately follows that if $f\left(a_{1}, \cdots, a_{n}\right) \subseteq g\left(a_{1}, \cdots, a_{n}\right)$ is a compositional tautology in $M$ then $f^{*}\left(Y_{a_{1}}, \cdots, Y_{a_{n}}\right) \subseteq g^{*}\left(Y_{a_{1}}, \cdots, Y_{a_{n}}\right)$ is a compositional tautology of $M / E$.

If $M$ is a multigroup and $Y$ is a normal Birkhoff subalgebra then

$$
\begin{aligned}
(Y \cdot a) \cdot(Y \cdot b) & =Y \cdot(a \cdot Y) \cdot b \\
& =Y \cdot(Y \cdot a) \cdot b=(Y \cdot Y) \cdot(a \cdot b)=Y \cdot(a \cdot b),
\end{aligned}
$$

and hence $E$ is an ideal for $M$. If $E$ is an ideal for a multiquasigroup $M$ then $(Y \cdot Y) \cdot(Y \cdot Y) \subseteq Y \cdot(Y \cdot Y)=Y \cdot Y$, so that $Y \cdot Y$ is a Birkhoff subalgebra. Since $Y \cdot Y \supseteqq Y$ it is clear that if $Y \cdot a \approx b$ then $(Y \cdot Y) \cdot a \approx b$. Suppose $(Y \cdot Y) \cdot a \approx b$. Then for some $x, x \cdot a \approx b$ and for some $y, Y \cdot y \approx x$. Since $a \equiv a(E)$ and $x \equiv y(E)$ and $x \cdot a \approx b$ there exists $c \equiv b$ such that $y \cdot a \approx c$. But then $a \equiv c \equiv b$, implying $Y \cdot a \approx b$. This completes the proof.

A further remark on the concept of compositional tautology may be clarifying. Although a relationship such as $(x \cdot x) \cdot y \leqq x \cdot y$ may be a tautology in $M$, the left-hand side is not a composition in the above 
sense. If the definition of composition is extended to allow identification of variables, as, e.g., $(x \cdot x) \cdot y$ is obtained from $(x \cdot u) \cdot y$ by identifying $u$ and $x$, it is easy to construct examples in which coideals do not preserve all compositions. However, ideals preserve even this broader class of compositions.

Let $E$ be an equivalence relation on $X$. If $A$ and $B$ are subsets of $X$ we shall write $A \equiv B(E)$ if and only if for every $x, A \approx x / E$ if and only if $B \approx x / E$. This defines an equivalence relation on the subsets of $X$. Clearly, if $E$ is an ideal for $M=(X, \cdot)$, and if $a \equiv$ $a_{1}(E), b \equiv b_{1}(E)$, also $a \cdot b \equiv a_{1} \cdot b_{1}(E)$. An element $x$ of $X$ such that, for every $a$ of $X, x \cdot a \equiv a \cdot x \equiv a(E)$ we call a congruent of $E$.

THEOREM 8. Let $Y$ be the set of all congruents of an ideal $E$ of a multiquasigroup $M=(X, \cdot)$. If $Y \neq \varnothing$, then
a. $(Y, \cdot)$ is a closed submultiquasigroup of $M$,
b. $Y$ is an equivalence class of $E$,
c. $M / E$ is a multiloop.

Further, if $E$ is a left congruence relation it is determined by $Y$, and $M / E$ is a regular decomposition.

Proof. If $x$ is in $Y$, and $y \equiv x$, then also $y$ is in $Y$, for if $x \cdot a \equiv a \cdot x \equiv a$ then also $y \cdot a \equiv a \cdot y \equiv a$, since $y \cdot a \equiv x \cdot a, a \cdot y \equiv a \cdot x$. If $x$ is in $Y, y$ in $Y$, then $x \equiv y$, for $x \equiv y \cdot x \equiv x \cdot y \equiv y$. Hence $Y$ is an equivalence class of $E$. If $x, y$ are in $Y$ and $z \approx x \cdot y \equiv x$ then $z \equiv x$ so $z$ is in $Y$. If $y, z$ are in $Y$ and $a$ solves $x \cdot y \approx z$ or $y \cdot x \approx z$ then $a$ is in $Y$ since $z \approx a \cdot y \equiv a$ or $z \approx y \cdot a \equiv a$ so $z \equiv a$. Therefore $(Y, \cdot)$ is a closed submultiquasigroup of $M$. If $A$ is in $X / E$ and $y, a$ are in $Y, A$ respectively, then if $b \approx y \cdot a \equiv a, b \equiv a$, so $Y \circ A \approx A$ in $M / E$. Similarly, $A \circ Y \approx A$ so $Y$ is a unit for $M / E$. Suppose that $E$ is a left congruence relation determined by $Z$, i.e., $a \equiv b(E)$ if and only if $Z \cdot a \approx b$. For $z$ in $Z, y$ in $Y$, if $z \cdot y \approx x$ then $y \equiv x \approx z \cdot y \equiv z$, showing $z$ is in $Y$. Hence, if $a \equiv b(E)$ then $Z \cdot a \approx b$ implying $Y \cdot a \approx b$. On the other hand, if $Y \cdot a \approx b$ then for some $y$ in $Y, y \cdot a \approx b$ implying $a \equiv y \cdot a \approx b$, so $a \equiv b$. Therefore $E$ is determined by $Y$, and from the remarks preceding Theorem 5 it follows that $M / E$ is a regular decomposition.

Finally, we shall generalize a theorem of Birkhoff [3, p. 86, Th. 2]. For $D$ and $E$ equivalence relations, $D \circ E=\{(x, z) \mid$ for some $y,(x, y) \in D$ and $(y, z) \in E\}$. If $D \circ E=E \circ D, D$ and $E$ are said to commute (or permute). Also, $D^{\prime}=\{(y, x) \mid(x, y) \in D\}$.

THEOREM 9. In a multiquasigroup a left congruence relation $D$ commutes with every ideal $E$. 
Proof. Let $Y$ be such that $a \equiv b(D)$ if and only if $Y \cdot a \approx b$. If $a \equiv x(D)$ and $x \equiv b(E)$ there exists $u$ in $Y$ such that $u \cdot x \approx a$. Since $u \equiv u(E)$ and $x \equiv b(E)$ and $u \cdot x \approx a$, there is some $v$ such that $u \cdot b \approx v$ and $v \equiv a(E)$. Since $u$ is in $Y$ and $u \cdot b \approx v, b \equiv v(D)$, and hence $(a, b) \in E \circ D$. Therefore, $D \circ E \subseteq E \circ D=E^{\prime} \circ D^{\prime}=(D \circ E)^{\prime}$. But for any binary relation $A$, if $A \subseteq A^{\prime}$ then $A=A^{\prime}$ since also $A^{\prime} \leqq\left(A^{\prime}\right)^{\prime}=A$. Hence $D \circ E=E \circ D$, showing that $D$ and $E$ commute.

5. Examples. Multiquasigroups are extremely abundant. To form such a system on a set $X$ it is only necessary to associate with each ordered pair $(a, b)$ in $X$ a nonvoid subset $A$ of $X$ in such a way that for every $a, \bigcup(a \cdot x)=X=\bigcup(x \cdot a)$, where $x$ runs over the members of $X$. If $X$ is finite such a system can be represented by an operation table in the usual fashion, the only novelty being the occurrence of multiple entries.

Example 1. Let $X=\{e, a, b, c, d\}$ and define "." by the following: table.

\begin{tabular}{cccccc}
\multicolumn{1}{c}{} & $e$ & $a$ & $b$ & $c$ & $d$ \\
\cline { 2 - 6 }$e$ & $e, a, b$ & $e, a, b$ & $e, a, b$ & $c, d$ & $c, d$ \\
$a$ & $a$ & $b$ & $e$ & $c, d$ & $e, a, b$ \\
$b$ & $b$ & $e$ & $a$ & $e, a, b$ & $c, d$ \\
$c$ & $e, c$ & $a, c$ & $b, c$ & $c$ & $d$ \\
$d$ & $e, d$ & $a, d$ & $b, d$ & $d$ & $c$
\end{tabular}

TABLE 1.

It is easily checked that $(X, \cdot)$ is a multiquasigroup which satisfies neither the left nor the right associative law, but has a unit element, namely $e$. It is therefore a multiloop. It has a single left coset decomposition determined by $Y=\{e\}$, with cosets $Y \cdot e=Y \cdot a=Y \cdot b=\{e, a, b\}$, $Y \cdot c=Y \cdot d=\{c, d\}$. The left congruence relation is a co-ideal but not an ideal.

ExAmple 2. Let $X$ be the set of complex numbers, and define $a \cdot b=\left\{x \mid x^{2}+a^{2}+b=0\right\}$. This system is a noncommutative, nonassociative multiquasigroup with neither right nor left units.

Multiquasigroups also arise in the following way. Let $(X, \cdot)$ be a multiquasigroup. A new multiquasigroup $(X, \circ)$ is defined by setting $x \circ y=\{a \mid a \cdot x \approx y\}$. This system might justifiably be called the left inverse of $(X, \cdot)$. Naturally, all multigroups and all quasigroups are multiquasigroups. Below we give a few examples of multigroups. These are also quite prevalent. 
EXAMPLE 3. Let $X_{\alpha}=\{\cos 0, \cos \pi / \alpha, \cos 2 \pi / \alpha, \cdots\}$ and define

$$
\cos m \pi / \alpha \cdot \cos n \pi / \alpha=\{\cos (m+n) \pi / \alpha, \cos (m-n) \pi / \alpha\} \text {. }
$$

The commutative multigroup $\left(X_{\alpha}, \cdot\right)$ has $\cos 0$ as a unit. If $\alpha$ is an integer $\left(X_{\alpha}, \cdot\right)$ is a finite multigroup with $|\alpha|+1$ elements. The operation table for $X_{3}=\{1,1 / 2,-1 / 2,-1\}$ is given in Table 2a. $X_{3}$ has a coset decomposition obtained by taking $Y=\{1,-1\}$, yielding $Y_{1}=$ $Y_{-1}=Y$ and $Y_{1 / 2}=Y_{-1 / 2}=\{1 / 2,-1 / 2\}$. The resulting homomorph is given in Table $2 b$. The corresponding equivalence relation is easily seen to be both an ideal and a co-ideal.

\begin{tabular}{c|cccc}
\multicolumn{1}{c}{} & 1 & $1 / 2$ & $-1 / 2$ & -1 \\
\cline { 2 - 5 } 1 & 1 & $1 / 2$ & $-1 / 2$ & -1 \\
$1 / 2$ & $1 / 2$ & $1,-1 / 2$ & $1 / 2,-1$ & $-1 / 2$ \\
$-1 / 2$ & $-1 / 2$ & $1 / 2,-1$ & $1,-1 / 2$ & $1 / 2$ \\
-1 & -1 & $-1 / 2$ & $1 / 2$ & 1
\end{tabular}

TABLE $2 \mathrm{a}$

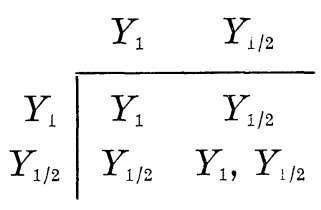

TABLE $2 b$

EXAMPLE 4. Let $(X, \wedge, \vee, \leqq)$ be a lattice and define $a \cdot b=$ $\{x \mid x \geqq a \wedge b\}, a \circ b=\{x \mid x \leqq a \vee b\}$. Both $(X, \cdot)$ and $(X, \circ)$ are commutative multigroups and every element is a unit. The only coset decomposition is determined by $X$, for if $Y$ determined a coset decomposition for $(X, \cdot)$, say, then if $a$ and $b$ are any two elements of $X$, $Y \cdot(a \wedge b)$ meets both $a$ and $b$. Hence they are in the same coset.

EXAMPLE 5. Let $X$ be a nonvoid open convex subset of the plane, and define $a \cdot b=\{x \mid x$ lies on the open line segment joining $a, b\}$ if $a \neq b$, and define $a \cdot a=a . \quad(X, \cdot)$ is a special case of a join system (Prenowitz [13]). In particular it is a commutative multigroup with no units. Many geometric concepts have algebraic analogs in this system. A convex subset $A$ of $X$ is a set such that if $x \in A$, $y \in A$ and $z$ lies on the join of $x$ and $y$ then $z \in A$, i.e., $x \cdot y \subseteq A$. Thus a convex subset is a Birkhoif subalgebra. An open convex subset $A$ also has the property that if $x \in A, y \in A$, then for some $z \in A, y$ lies on the join of $z$ and $x$, i.e., $y \approx z \cdot x$. Therefore an open convex subset is a submultigroup. A linear section $L$ of $X$ is subset holding, with each pair $x, y$ in $L$, any point in $X$ in the line on which they lie. $L$ therefore holds $x \cdot y$, and all solutions to $a \cdot x \approx y, x \cdot b \approx y$. Hence $L$ is a closed submultigroup. Conversely, any closed submultigroup is either $X$, a linear section, or $\varnothing$.

Because $b \cdot a \approx a$ in a join system only if $b=a$, if $Y \cdot a \approx a$ for every $a$ in $X$ then $Y=X$. Hence join systems have no proper left 
coset decompositions. In the present example consider the decomposition of $X$ consisting of a point $a$ in $X$, and the open ray segments extending from $a$, lying in $X$. This is an example of an ideal, the resulting homomorph being a multigroup with a unit and unique inverses for every element. Geometrically it is analogous to a circle with its center point. One verifies easily that the set of congruents of this ideal is $\{a\} \neq \varnothing$.

Example 6. Let $X=\{e, a, b\}$ and define $x \circ_{1} y, x \circ_{2} y$, and $x \circ_{3} y$ by Tables $3,4,5$, respectively. The multigroups $\left(X, \circ_{1}\right),\left(X, \circ_{2}\right),\left(X, \circ_{3}\right)$ are respectively commutative with no unit, commutative with a unit, and noncommutative with a unit. H. Campaigne [6] has shown that $\left(X, \circ_{1}\right)$ is not a homomorph of any group.

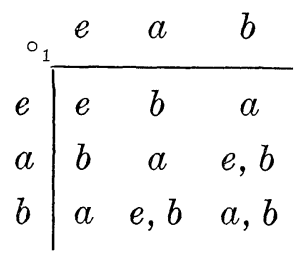

TABLE 3

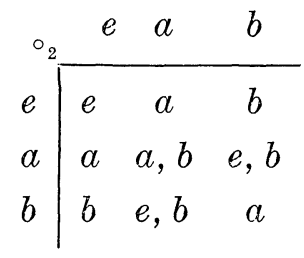

TABLE 4

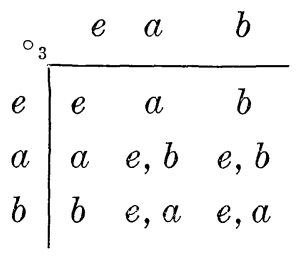

TABLE 5

ExAMPLE 7. Take $X$ to be the dihedral group having generators $a$ and $b$ satisfying $a^{4}=b^{2}=e, b a=a^{3} b$. The subgroup $\{e, b\}$ determines the left coset decomposition $\left\{\{e, b\},\{a, a b\},\left\{a^{2}, a^{2} b\right\},\left\{a^{3}, a^{3} b\right\}\right\}$. Under the operation defined in $\S 4$ these cosets form a multigroup with the operation Table 6 . The results of $\S 2$ and $\S 4$ show that the equivalence relation associated with the decomposition is a co-ideal but not an ideal.

\begin{tabular}{|c|c|c|c|c|}
\hline & $\{e, b\}$ & $\{a, a b\}$ & $\left\{a^{2}, a^{2} b\right\}$ & $\left\{a^{3}, a^{3} b\right\}$ \\
\hline$\{e, b\}$ & $\{e, b\}$ & $\begin{array}{l}\{a, a b\} \\
\left\{a^{3}, a^{3} b\right\}\end{array}$ & $\left\{a^{2}, a^{2} b\right\}$ & $\begin{array}{l}\{a, a b\} \\
\left\{a^{3}, a^{3} b\right\}\end{array}$ \\
\hline$\{a, a b\}$ & $\{a, a b\}$ & $\begin{array}{l}\{e, b\}, \\
\left\{a^{2}, a^{2} b\right\}\end{array}$ & $\left\{a^{3}, a^{3} b\right\}$ & $\begin{array}{l}\{e, b\}, \\
\left\{a^{2}, a^{2} b\right\}\end{array}$ \\
\hline$\left\{a^{2}, a^{2} b\right\}$ & $\left\{a^{2}, a^{2} b\right\}$ & $\begin{array}{l}\{a, a b\}, \\
\left\{a^{3}, a^{3} b\right\}\end{array}$ & $\{e, b\}$ & $\begin{array}{l}\{a, a b\} \\
\left\{a^{3}, a^{3} b\right\}\end{array}$ \\
\hline$\left\{a^{3}, a^{3} b\right\}$ & $\left\{a^{3}, a^{3} b\right\}$ & $\begin{array}{l}\{e, b\} \\
\left\{a^{2}, a^{2} b\right\}\end{array}$ & $\{a, a b\}$ & $\begin{array}{l}\{e, b\} \\
\left\{a^{2}, a^{2} b\right\}\end{array}$ \\
\hline
\end{tabular}




\section{REFERENCES}

1. M. Benado, Asupra unei probleme a lui Garrett Birkhoff, Acad. Rep. Pop. Rom. Bul. Sci. 6 (1954), 703-730.

2. - Les ensembles partiellement ordoneés et le théorème de raffinement de Schreier, II., Czechoslovakian Mathematical Journal 80 (1955), 308-343.

3. G. Birkhoff, Lattice theory, Amer. Math. Soc. Coll. Publ., Revised Edition, 1948.

4. R. Bruck, A survey of binary systems, Ergebnisse der Mathematik und ihrer Grenzgebiete, Springer-Verlag, 1958.

5. P. Brunovský, O zovšeobeenenýeh algebraickýeh systémoch, Acta Fac. Rerum nat. univ. com. math. 111 (1958), 41-54.

6. H. Campaigne, Partition hypergroups, Amer. J. Math. 62 (1940), 599-612.

7. M. Dresher and O. Ore, Theory of multigroups, Amer. J. Math. 60 (1938), 705-733.

8. M. Krasner, Hypergroupes extramoduliformes et moduliformes, C. R. Acad. Sci. (Paris) 219 (1944), 473-476.

9. F. Marty, Sur les groupes et hypergroupes, Ann. de l'Ecole Normale 53 (1936), 83-123.

10. R. Pate, Rings with multiple-valued operations, Amer. J. Math. 64 (1942), 506-517.

11. G. Pickert, Bemerkungen zum Homomorphiebegriff, Math. Zeit. 53 (1950), 375-386.

12. H. Pickett, Subdirect representations of relational systems, Fund. Math. 56 (1964), 223-240.

13. W. Prenowitz, Projective geometries as multigroups, Amer. J. Math. 65 (1943), 235-256.

14. — Descriptive geometries as multigroups, Trans, Amer. Math., Soc. 59 (1946), 333-380.

15.

Spherical geometries and multigroups, Canad. J. Math. 2 (1950), 100-119.

Received June 9, 1965.

Chevron Research Company

RICHMOND, CALIFORNIA 


\section{PACIFIC JOURNAL OF MATHEMATICS}

\section{EDITORS}

H. SAMELSON

Stanford University

Stanford, California

J. P. JANS

University of Washington

Seattle, Washington 98105

\section{J. DugunduI}

University of Southern California Los Angeles, California 90007

RICHARD ARENS

University of California

Los Angeles, California 90024

\section{ASSOCIATE EDITORS}
E. F. BECKENBACH
B. H. NEUMANN
F. WOLF
K. YoSIDA

\section{SUPPORTING INSTITUTIONS}

\author{
UNIVERSITY OF BRITISH COLUMRIA \\ CALIFORNIA INSTITUTE OF TECHNOLOGY \\ UNIVERSITY OF CALIFORNIA \\ MONTANA STATE UNIVERSITY \\ UNIVERSITY OF NEVADA \\ NEW MEXICO STATE UNIVERSITY \\ OREGON STATE UNIVERSITY \\ UNIVERSITY OF OREGON \\ OSAKA UNIVERSITY \\ UNIVERSITY OF SOUTHERN CALIFORNIA
}

\author{
STANFORD UNIVERSITY \\ UNIVERSITY OF TOKYO \\ UNIVERSITY OF UTAH \\ WASHINGTON STATE UNIVERSITY \\ UNIVERSITY OF WASHINGTON \\ AMERICAN MATHEMATICAL SOCIETY \\ CHEVRON RESEARCH CORPORATION \\ TRW SYSTEMS \\ NAVAL ORDNANCE TEST STATION
}

Mathematical papers intended for publication in the Pacific Journal of Mathematics should be typewritten (double spaced). The first paragraph or two must be capable of being used separately as a synopsis of the entire paper. It should not contain references to the bibliography. Manuscripts may be sent to any one of the four editors. All other communications to the editors should be addressed to the managing editor, Richard Arens at the University of California, Los Angeles, California 90024 .

50 reprints per author of each article are furnished free of charge; additional copies may be obtained at cost in multiples of 50 .

The Pacific Journal of Mathematics is published monthly. Effective with Volume 16 the price per volume (3 numbers) is $\$ 8.00$; single issues, $\$ 3.00$. Special price for current issues to individual faculty members of supporting institutions and to individual members of the American Mathematical Society: $\$ 4.00$ per volume; single issues $\$ 1.50$. Back numbers are available.

Subscriptions, orders for back numbers, and changes of address should be sent to Pacific Journal of Mathematics, 103 Highland Boulevard, Berkeley 8, California.

Printed at Kokusai Bunken Insatsusha (International Academic Printing Co., Ltd.), No. 6, 2-chome, Fujimi-cho, Chiyoda-ku, Tokyo, Japan.

\section{PUBLISHED BY PACIFIC JOURNAL OF MATHEMATICS, A NON-PROFIT CORPORATION}

The Supporting Institutions listed above contribute to the cost of publication of this Journal, but they are not owners or publishers and have no responsibility for its content or policies. 


\section{Pacific Journal of Mathematics}

\section{Vol. 21, No. 2 December, 1967}

Arne P. Baartz, The measure algebra of a locally compact semigroup ..... 199

Robert F. Brown, On maps with identical fixed point sets............. 215

C. Buttin, Existence of a homotopy operator for Spencer's sequence in the analytic case ..................................... 219

Henry Werner Davis, An elementary proof that Haar measurable almost periodic functions are continuous ........................ 241

Zeev Ditzian, On asymptotic estimates for kernels of convolution transforms ...................................... 249

Robert E. Edwards, Boundedness principles and Fourier theory ......... 255

John A. Hildebrant, On compact unithetic semigroups ............... 265

Marinus A. Kaashoek and David Clark Lay, On operators whose Fredholm set is the complex plane ............................ 275

Sadao Kató, Canonical domains in several complex variables ........... 279

David Clifford Kay, The ptolemaic inequality in Hilbert geometries.... . . . 293

Joseph D. E. Konhauser, Biorthogonal polynomials suggested by the Laguerre polynomials ............................. 303

Kevin Mor McCrimmon, Macdonald's theorem with inverses .......... 315

Harry Eldon Pickett, Homomorphisms and subalgebras of multialgebras .................................... 327

Richard Dennis Sinkhorn and Paul Joseph Knopp, Concerning nonnegative matrices and doubly stochastic matrices ..............

Erling Stormer, On anti-automorphisms of von Neumann algebras ...

Miyuki Yamada, Regular semi-groups whose idempotents satisfy permutation identities .......................... 